\title{
Efecto del riego deficitario en la acumulación de compuestos antioxidantes en plantas de tomate
}

\section{Effect of deficit irrigation on the accumulation of antioxidant compounds in tomato plants}

\begin{abstract}
José Refugio Méndez-Vázquez 1 (D)
Adalberto Benavides-Mendoza 2 (D)

Antonio Juárez-Maldonado ${ }^{3}$ (D)

Marcelino Cabrera-De la Fuente ${ }^{2}$ [D

Armando Robledo-Olivo 4 (D)

Susana González-Morales ${ }^{5 *}$ [D

${ }^{1}$ Doctorado en Ciencias en Agricultura

Protegida, Universidad Autónoma

Agraria Antonio Narro. Calzada An-

tonio Narro 1923, CP. 25315. Saltillo,

Coahuila, México.

${ }^{2}$ Departamento de Horticultura, Uni-

versidad Autónoma Agraria Antonio

Narro. Calzada Antonio Narro 1923,

CP. 25315. Saltillo, Coahuila, México.

${ }^{3}$ Departamento de Botánica, Universi-

dad Autónoma Agraria Antonio Narro.

Calzada Antonio Narro 1923, CP.

25315. Saltillo, Coahuila, México.

${ }^{4}$ Departamento de Ciencia y Tec-

nología en Alimentos, Universidad

Autónoma Agraria Antonio Narro.

Calzada Antonio Narro 1923, CP.

25315. Saltillo, Coahuila, México.

${ }^{5}$ CONACYT- Universidad Autónoma

Agraria Antonio Narro. Calzada An-

tonio Narro 1923, CP. 25315. Saltillo,

Coahuila, México.

*Autor de correspondencia:

qfb_sgm@hotmail.com;

sgonzalezmo@conacyt.mx
\end{abstract}

\section{Nota científica}

Recibida: 29 de enero 2021

Aceptada: 11 de mayo 2021

Como citar: Méndez-Vázquez JR, Benavides-Mendoza A, JuárezMaldonado A, Cabrera-De la Fuente M, Robledo-Olivo A, González-Morales $S$ (2021) Efecto del riego deficitario en la acumulación de compuestos antioxidantes en plantas de tomate. Ecosistemas y Recursos Agropecuarios 8(2): e2822. DOI: 10.19136/era.a8n2.2822
RESUMEN. El uso eficiente del agua en la agricultura es la clave para mitigar la escasez de los recursos hídricos. El riego deficitario controlado (RDC) es una estrategia utilizada en la cual se reduce el aporte de agua sin afectar la producción y calidad de la cosecha. El objetivo del trabajo fue evaluar el efecto en el metabolismo antioxidante en la etapa de floración y producción en plantas de tomate bajo dos niveles de RDC (50 y 75\%) durante el ciclo del cultivo. Los resultados muestran un incremento en la actividad antioxidante y actividad ascorbato peroxidasa, y disminución de la actividad catalasa en floración en los tratamientos con RDC, mientras que en producción los tratamientos con RDC disminuyeron en contenido de proteínas totales y la capacidad antioxidante. Las plantas de tomate bajo RDC modificaron el metabolismo antioxidante para mitigar el daño oxidativo por el déficit de agua.

Palabras clave: Ascorbato peroxidasa, capacidad antioxidante, catalasa, uso eficiente del agua.

ABSTRACT. Efficient use of water in agriculture is the key to mitigating scarcity of water resources. Deficit irrigation (DI) is a strategy used that reduces the water supply without affecting the production and quality of the harvest. The objective of this work was to evaluate the effect on antioxidant metabolism in the flowering and production stage of tomato plants under two levels of DI (50 and 75\%) based on evapotranspiration by the gravimetric method during the cultivation cycle. The results showed an increase in the antioxidant activity and ascorbate peroxidase activity in flowering in the treatments with $\mathrm{DI}$, while in production the treatments with $\mathrm{DI}$ decreased the total proteins and the antioxidant activity. Tomato plants under DI modified antioxidant metabolism to mitigate oxidative damage due to water deficit.

Key words: Ascorbate peroxidase, antioxidant capacity, catalase, efficient use of water. 


\section{INTRODUCCIÓN}

La falta de agua es uno de los principales problemas a los que se enfrenta la humanidad, aunado al cambio climático, las sequias prolongadas y el aumento de la población que continuarán provocando una presión sobre este valioso recurso natural en las zonas áridas y semiáridas (Sweet et al. 2017, Bigurra-Alzati et al. 2021). A nivel mundial el sector agrícola es el mayor consumidor de agua, ya que representa aproximadamente el $70 \%$ del uso de agua dulce, comparado con un $20 \%$ de uso industrial y un $10 \%$ de uso doméstico (FAO 2016). Por lo anterior, es necesario considerar estrategias de gestión del agua para la actividad agrícola. El riego deficitario controlado (RDC) es una estrategia clave para el ahorro del agua en la agricultura, el cual consiste en la aplicación del riego por debajo de la evapotranspiración completa del cultivo, sin llegar a causar daños por estrés hídrico (Chai et al. 2014, Rady et al. 2020). En México, el RDC es una técnica que se ha venido aplicando en una variedad de cultivos. Pero se tiene falta de comprensión de los mecanismos con los que las plantas responden al RDC, específicamente se conoce poco sobre como el RDC puede aumentar la productividad de los cultivos al mismo tiempo que reduce la cantidad de agua de riego en la agricultura (Chai et al. 2016).

El déficit hídrico reduce el potencial hídrico en el suelo, afectando los procesos de transpiración, fotosíntesis, apertura de estomas, temperatura de las hojas y el metabolismo antioxidante; por lo que se ve afectado el crecimiento, desarrollo y rendimiento de los cultivos de importancia económica (Nawaz et al. 2015, Toor et al. 2020). Lo anterior genera un desequilibrio en el metabolismo fotosintético, provocando incremento en la producción de especies reactivas de oxígeno (ERO) (Taha et al. 2020). Las principales ERO que se generan son el oxígeno singlete $\left({ }_{1} \mathrm{O}^{2}\right)$, súper óxido $\left(\mathrm{O}_{2^{-}}\right)$, radical hidroxilo $(\mathrm{OH})$ y peróxido de hidrogeno $\left(\mathrm{H}_{2} \mathrm{O}_{2}\right)$ (Dvorak et al. 2020). Las ERO son generadas en los cloroplastos, mitocondrias y peroxisomas, siendo estos organelos los principales afectados ante el déficit hídrico (Corpas et al. 2019). El aumento constante de las ERO provocan daños en las células como la peroxidación lipídica de las paredes celulares, la destrucción del aparato fotosintéticos y daños al ADN, causando estrés oxidativo celular (Koffler et al. 2014). Ante esta situación, las plantas han desarrollado mecanismos para mitigar el estrés oxidativo causado por la generación excesiva de ERO, a través del metabolismo antioxidante. El cual está conformado por antioxidantes no enzimáticos como el glutatión (GSH), ácido ascórbico y carotenoides, así como los antioxidantes enzimáticos como la superóxido dismutasa (SOD), catalasa (CAT), ascorbato peroxidasa (APX), entre otras (He et al. 2018, Shukla et al. 2019). Los antioxidantes enzimáticos y no enzimáticos conforman el sistema de defensa antioxidante de las plantas al encabezar la primera y segunda línea de defensa para la eliminación y regulación de la concentración de las ERO (Haida y Hakiman 2019).

El estudio del metabolismo antioxidante en plantas bajo RDC pretende contribuir en explicar el mecanismo de adaptación de las plantas, particularmente el cómo se podría incrementar la producción de cultivos bajo un régimen de RDC. Por lo anterior el objetivo del presente trabajo consistió en evaluar la producción de compuestos relacionados al metabolismo antioxidante en plantas de tomate bajo dos niveles de RDC (50 y 75\%) durante el ciclo del cultivo.

\section{MATERIALES Y MÉTODOS}

\section{Establecimiento del cultivo y tratamientos}

El cultivo se desarrolló en un invernadero con cubierta de polietileno con un $70 \%$ de irradiancia natural, donde se evaluó el efecto de dos tratamientos de RDC en el híbrido comercial de tomate El Cid F1 (Harris Moran) de crecimiento indeterminado. Se utilizaron macetas de $10 \mathrm{~L}$ de capacidad con sustrato peat-moss y perlita en una relación 1:1 (v/v). Los tratamientos consistieron en la reposición de agua pérdida por la evapotranspiración por el método gravimétrico (Segura-Castruita et al. 2011) en un 50 (severo), 75 (moderado) y 100\% (control), mediante la siguiente fórmula:

$$
\text { Evapotranspiración }=P 1-P 2
$$


Donde: $\mathrm{P} 1$ es el peso de la maceta 15 minutos después de haberse saturado con agua y dejado drenar y P2 es el peso de la maceta transcurridas 24 $\mathrm{h}$.

Las mediciones se realizaron alrededor de las 7 y $8 \mathrm{~h}$ de la mañana, antes de que comenzara la evapotranspiración, ya que ésta representa la pérdida de agua por la transpiración de la planta y la pérdida de agua del sustrato por la evaporación.

Para la nutrición de las plantas se utilizó una solución Steiner (Steiner 1961) de acuerdo con su etapa fenológica (25\% en etapa vegetativa, $50 \%$ en floración y $100 \%$ en producción de frutos).

El diseño experimental utilizado fue completamente al azar con 20 repeticiones. La unidad experimental fue una planta en una maceta. Se realizaron dos muestreos en las hojas de las plantas, uno en la etapa de floración a los 30 días después del trasplante (DDT) y otro en la etapa de producción de frutos a los 90 DDT. Se recolectó la cuarta hoja fisiológicamente madura de cada tratamiento, las cuales se congelaron a $-80^{\circ} \mathrm{C}$, y posteriormente se liofilizaron en un Free Zone modelo $2.5 \mathrm{~L}$ (Labconco).

\section{Análisis Bioquímico}

Se midió el contenido de proteína total (mg $\mathrm{g}^{-1}$ peso seco PS) con la metodología de Bradford (1976). El contenido de GSH ( $\mathrm{mg} \mathrm{g}^{-1}$ PS) se determinó con la metodología descrita por Xue et al. (2001) a través de la reacción con el ácido 5,5ditio-bis-2 nitrobenzoico (DTNB). La capacidad antioxidante total se determinó por el método descrito por Re et al. (1999) mediante el radical ABTS (mg de ácido ascórbico $\mathrm{g}^{-1}$ PS). Para los análisis anteriores se evaluaron seis repeticiones, cada repetición constó de las hojas de una planta por tratamiento en cada etapa de muestreo.

\section{Actividad enzimática}

La actividad catalasa (CAT) (EC 1.11.1.6) se determinó con la metodología de Cansev et al. (2011) y se expresó como $U$ por gramo de proteínas totales $\left(\mathrm{U} \mathrm{g} \mathrm{g}^{-1} \mathrm{PT}\right)$, donde $\mathrm{U}$ es igual a $\mathrm{mM}$ de $\mathrm{H}_{2} \mathrm{O}_{2}$ consumido por mililitro por minuto. La actividad de glutatión peroxidasa (GPX) (EC 1.11.1.9) se deter- minó según Xue et al. (2001) y los resultados se expresaron en $U$ por gramo de proteínas totales $\left(\mathrm{U} \mathrm{g} \mathrm{g}^{-1} \mathrm{PT}\right)$, donde $\mathrm{U}$ es igual a $\mathrm{mM}$ de $\mathrm{GSH}$ por mililitro por minuto. La actividad ascorbato peroxidasa (APX) (EC 1.11.1.11) se midió según Nakano y Asada (1987) y se expresó como U por gramo de proteínas totales $\left(\mathrm{U} \mathrm{g}^{-1} \mathrm{PT}\right)$, donde $\mathrm{U}$ es igual a $\mu \mathrm{mol}$ de ascorbato oxidado por mililitro por minuto. Para los análisis anteriores se evaluaron seis repeticiones, cada repetición constó de las hojas de una planta por tratamiento por cada etapa de muestreo.

\section{Análisis estadístico}

Para analizar los resultados obtenidos, se realizó un análisis de varianza y una prueba de medias de la diferencia mínima significativa (LSD) de Fisher $(p \leq 0.05)$ utilizando el software Infostat 2016.

\section{RESULTADOS Y DISCUSIÓN}

\section{Análisis bioquímico}

El contenido de proteínas totales en la etapa de floración no presentó diferencias significativas entre tratamientos; sin embargo, en la etapa de producción el tratamiento de RDC $50 \%$ presentó una disminución $(p \leq 0.05)$ del $16.81 \%$ con respecto al tratamiento control (Tabla 1). En el contenido de GSH en la etapa floración el tratamiento de RDC 50\% incrementó ( $\mathrm{p} \leq 0.05$ ) un $11.98 \%$ con respecto al tratamiento de RDC $75 \%$; pero ambos tratamientos con RDC fueron similares al testigo. En la etapa de producción no se presentaron diferencias significativas entre tratamientos en el contenido de GSH.

En la etapa de floración el tratamiento de RDC $50 \%$ presentó un incremento $(\mathrm{p} \leq 0.05)$ del $25.12 \%$ en la capacidad antioxidante total en comparación con el control. Pero en la etapa de producción ambos tratamientos con RDC (50 y 75\%) presentaron una disminución $(p \leq 0.05)$ del 24.58 y $29.81 \%$ con respecto al control.

Los efectos por el déficit hídrico se pueden manifestar en las diferentes etapas del desarrollo de las plantas y en diferentes niveles dependiendo del grado de restricción hídrica al que se sometan (Saed-Moucheshi et al. 2014). Una de las primeras 
Tabla 1. Contenido de proteínas totales, glutatión reducido y capacidad antioxidante total en hojas tomate bajo dos niveles de RDC.

\begin{tabular}{ccccc}
\hline \multirow{2}{*}{$\begin{array}{c}\text { Etapa } \\
\text { fenológica }\end{array}$} & \multirow{2}{*}{ Tratamiento } & PT & GSH & CA \\
\cline { 3 - 5 } & & $\left(\mathrm{mg} \mathrm{g}^{-1} \mathrm{PS}\right)$ & $\left(\mathrm{mg} \mathrm{g}^{-1} \mathrm{PS}\right)$ & $\left(\mathrm{mg} \mathrm{ASA} \mathrm{g}^{-1} \mathrm{PS}\right)$ \\
\hline \multirow{3}{*}{ Floración } & Control 100\% & $74.25^{a}$ & $2.92^{a b}$ & $21.69^{b}$ \\
& $\mathrm{RDC} 75 \%$ & $62.48^{a}$ & $2.82^{b}$ & $22.28^{b}$ \\
& $\mathrm{RDC} 50 \%$ & $79.73^{a}$ & $3.17^{a}$ & $27.14^{a}$ \\
\multirow{5}{*}{ Producción } & Control 100\% & $93.67^{a}$ & $3.06^{a}$ & $28.11^{a}$ \\
& $\mathrm{RDC} \mathrm{75 \%}$ & $86.23^{a b}$ & $3.0^{a}$ & $19.73^{b}$ \\
& $\mathrm{RDC} \mathrm{50 \%}$ & $77.92^{b}$ & $3.01^{a}$ & $21.2^{b}$ \\
\hline
\end{tabular}

Medias por columna seguidas con la misma letra son estadísticamente iguales de acuerdo a LSD $(p \leq 0.05)$. PT: proteínas totales, CA: capacidad antioxidante total, GSH: glutatión reducido, ASA: ácido ascórbico, PS: peso seco.

respuestas de las plantas al déficit hídrico es el ajuste osmótico, el cual es un mecanismo esencial para la tolerancia a la falta de agua, siendo la acumulación de proteínas y de osmolitos una respuesta celular que tiene la finalidad de detener la pérdida de agua al aumentar la presión osmótica, de tal manera que se logre evitar la pérdida de la turgencia celular (Xu et al. 2020). Pero cuando existe una restricción hídrica severa, se puede afectar la tasa de fotosíntesis en los cultivos al reducir el contenido de proteína soluble en las hojas bandera (Gupta et al. 2011).

Una de las consecuencias de estar bajo restricción hídrica es el cierre estomático, por lo que las plantas disminuyen la tasa de fijación de $\mathrm{CO}_{2}$ provocando un incremento en la producción de ERO (Agami et al. 2018). Por lo anterior, el sistema antioxidante es el primero en intervenir, algunos de los compuestos antioxidantes más importantes son los compuestos fenólicos, carotenoides, ácido ascórbico y el GSH (Chai et al. 2016). La importancia del GSH radica en que actúa en el ciclo del glutatión-ascorbato, el cual es de gran importancia en la defensa antioxidante para la detoxificación eficiente de ERO y peróxidos orgánicos en las plantas, ayudando a proteger el aparato fotosintético del daño oxidativo debido al déficit hídrico (Xie et al. 2019, Melandri et al. 2019). Además, desempeña funciones importantes en diversos procesos biológicos incluidos la regulación del transporte de azufre, síntesis de fitoquelatinas, detoxificación de xenobióticos, transducción de señales, crecimiento y desarrollo celular y expresión de genes susceptibles de estrés (Bartoli et al. 2017). El estrés oxidativo se desen- cadena cuando se interrumpe el equilibrio entre la acumulación de ERO y los sistemas de captación de radicales libres (antioxidantes), lo cual provoca un desequilibrio en la planta (Nahar et al. 2015). Las plantas cuentan con mecanismos para combatir condiciones adversas y prevenir daños nocivos (Qi et al. 2018), siendo el sistema antioxidante una de las formas más comunes en como las plantas resisten el estrés. Debido a la gran variedad de compuestos antioxidantes, las propiedades antioxidantes celulares se caracterizan usualmente como capacidad antioxidante total (Csepregi et al. 2016).

\section{Actividad enzimática}

La actividad enzimática de APX en la etapa de floración presentó diferencias significativas $(p \leq$ 0.05 ) entre los tratamientos con RDC 50 y $75 \%$ (Figura 1), los cuales aumentaron un 120 y $100 \%$, respectivamente, en comparación con el control; mientras que en la etapa de producción no se presentaron diferencias significativas. Para la actividad enzimática de CAT (Figura 2) en la etapa de floración se presentaron diferencias significativas $(p \leq 0.05)$ en los tratamientos con RDC 50 y $75 \%$, los cuales disminuyeron un 24.69 y $33.53 \%$, respectivamente, en comparación con el control; aunque este efecto no se observó en la etapa de producción. Por otro lado, para la actividad enzimática de GPX (Figura 3) no se presentaron diferencias significativas entre tratamientos.

Los antioxidantes enzimáticos juegan un papel esencial en la homeostasis del $\mathrm{H}_{2} \mathrm{O}_{2}$ previniendo el daño oxidativo celular (Ren et al. 2020). La variación 


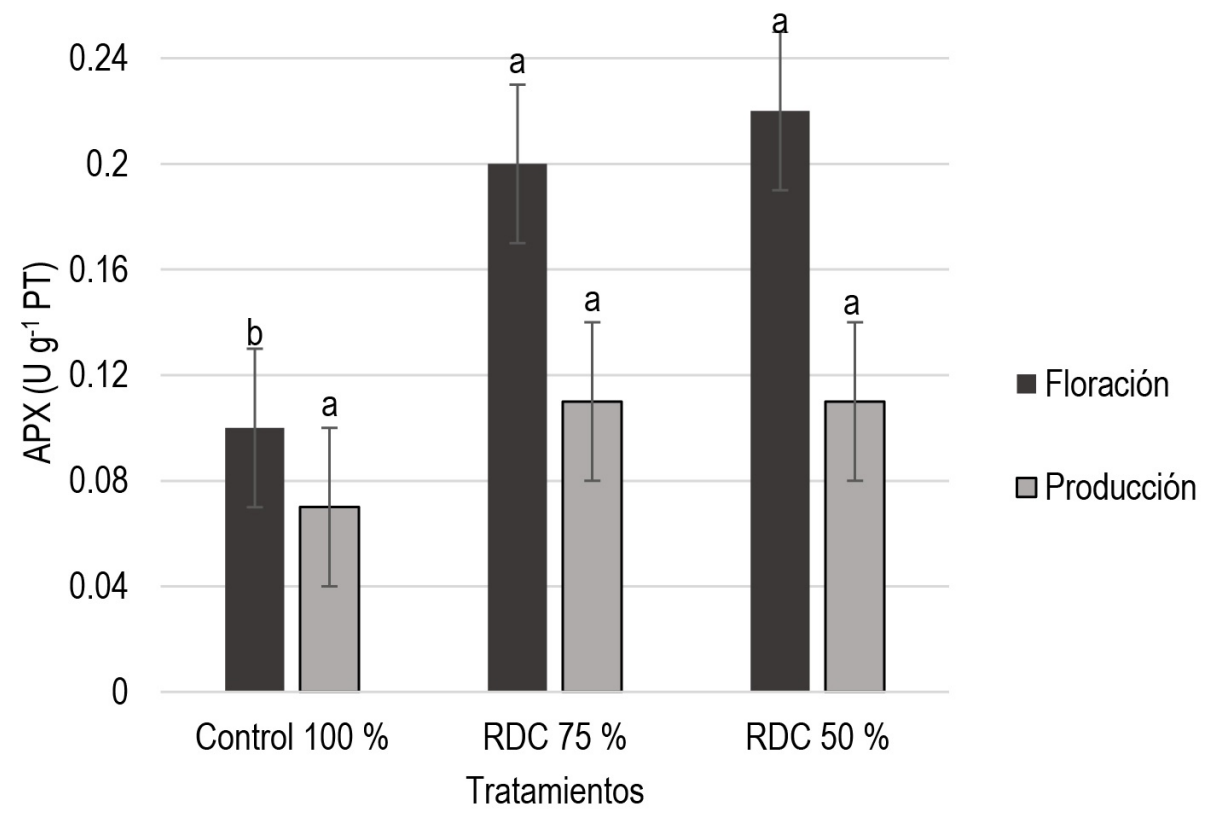

Figura 1. Actividad enzimática de ascorbato peroxidasa en hojas tomate bajo dos niveles de RDC. Letras diferentes en las barras indican diferencias significativas entre tratamientos de acuerdo con LSD $(p \leq$ 0.05).

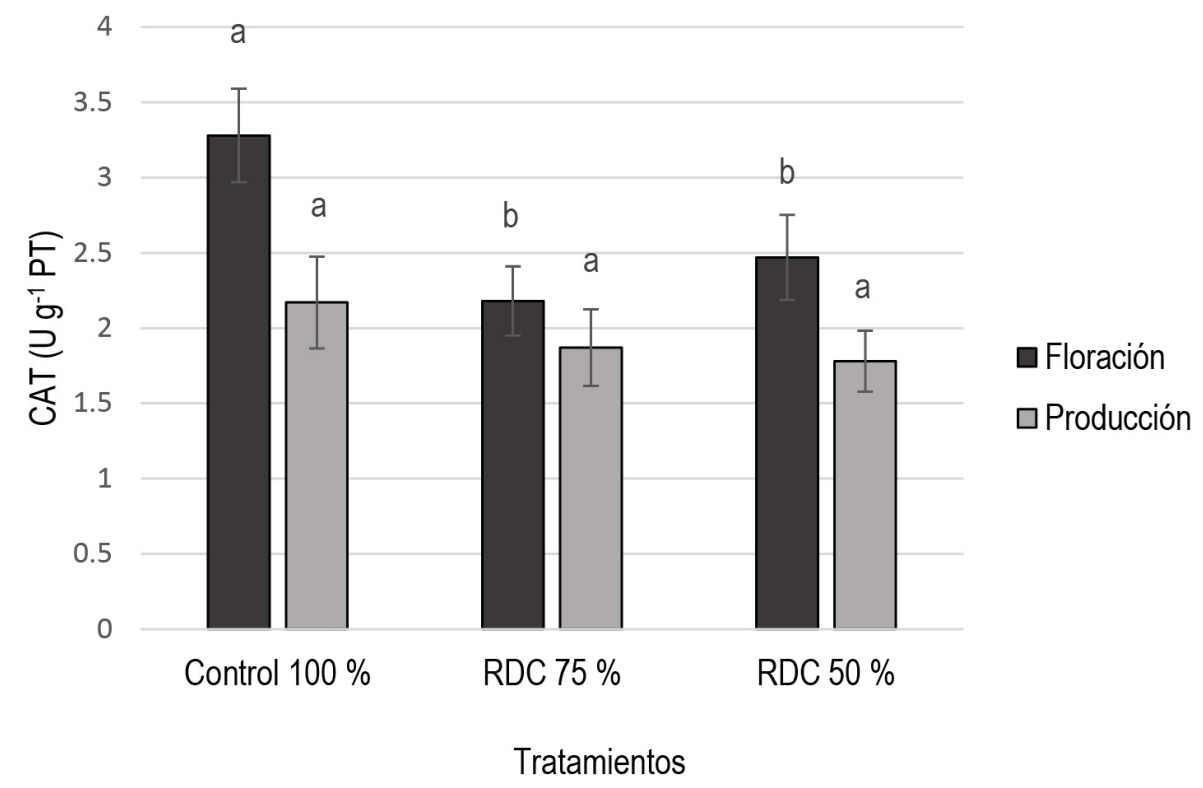

Figura 2. Actividad enzimática de catalasa en hojas tomate bajo dos niveles de RDC. Letras diferentes en las barras indican diferencias significativas entre tratamientos de acuerdo con LSD $(p \leq 0.05)$.

en la actividad de enzimas antioxidantes es posiblemente la ruta principal para tolerar el estrés hídrico en las plantas (Denaxa et al. 2020). Tanto APX como CAT y GPX representan los principales eliminadores de $\mathrm{H}_{2} \mathrm{O}_{2}$ en las plantas (Laxa et al. 2019), las cuales se encuentran distribuidas en la mayoría de los organelos vegetales siendo los cloroplastos, mitocondrias y peroxisomas donde se encuentran la mayor actividad de éstas (Meitha et al. 2020). La enzima CAT es clave en la captación catalítica del 


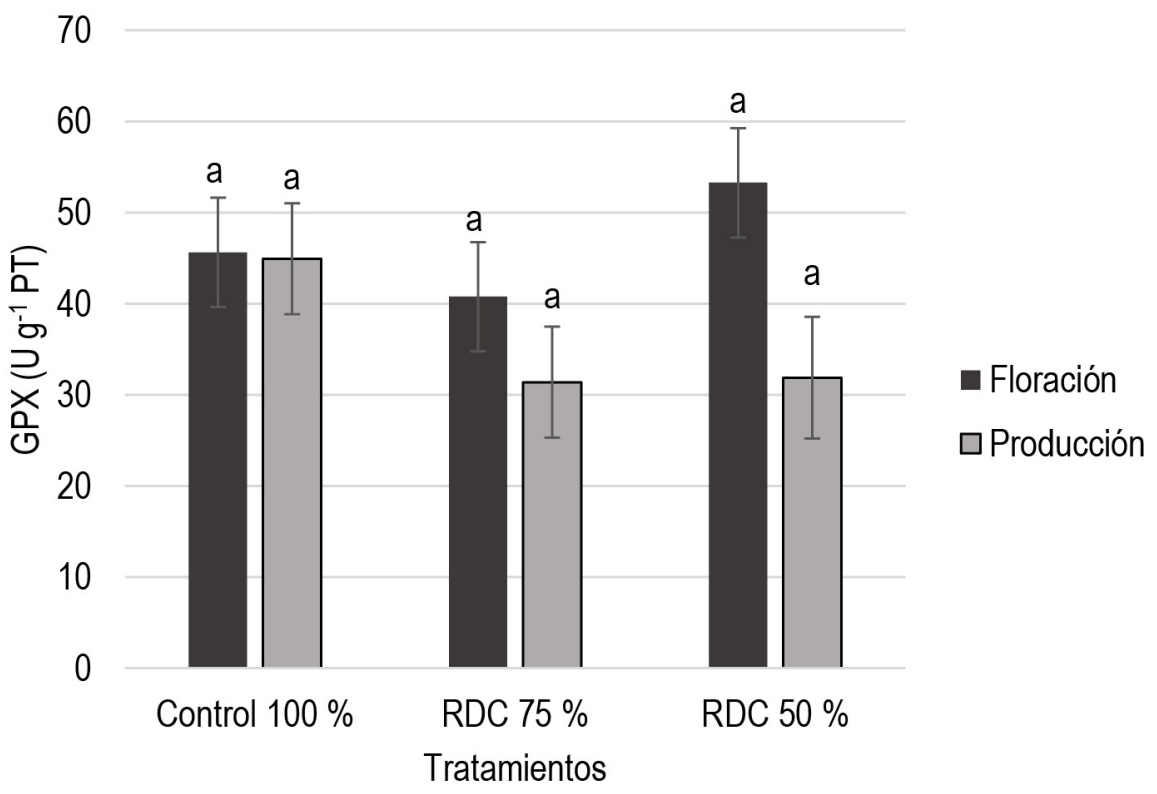

Figura 3. Actividad enzimática de glutatión peroxidasa en hojas tomate bajo dos niveles de RDC. Letras diferentes en las barras indican diferencias significativas entre tratamientos de acuerdo con LSD $(p \leq 0.05)$.

$\mathrm{H}_{2} \mathrm{O}_{2}$ en agua y oxígeno (Leung 2018), generalmente aumenta la actividad cuando las plantas se encuentran en condiciones adversas de crecimiento; pero algunos estudios reportan que disminuye su actividad bajo condiciones de estrés, por lo que es cuestionable considerarla como un marcador de estrés (Leung 2018).

La enzima APX participa en el metabolismo activo del oxígeno en las plantas, catalizando la formación de $\mathrm{O}_{2}$ y $\mathrm{H}_{2} \mathrm{O}$ a partir de $\mathrm{H}_{2} \mathrm{O}_{2}$ para proteger a la planta del daño oxidativo (Leng et al. 2021). En dos genotipos de frijol (Phaseolus vulgaris L.) bajo condiciones de riego deficitario se reporta el incremento del contenido de enzimas antioxidantes como APX, SOD, CAT y peroxidasa (POD) (Soureshjani et al. 2019). Al disminuir la cantidad de agua suministrada en etapas tempranas se pudo observar un incremento en la actividad APX. Lo que sugiere que la aplicación del RDC desde etapas tempranas ayuda a realizar las modificaciones metabólicas necesarias para lograr el equilibrio en el balance de ERO y antioxidantes. Al respecto, estudios realizados bajo diferentes condiciones de RDC reportan que la aplicación del déficit hídrico ayuda a equilibrar el metabolismo donde la generación de ERO disminuye, ya que las plantas logran regularizar la captación de $\mathrm{CO}_{2}$, por lo que la actividad antioxidante no se ve alterada (Nangare et al. 2016, Noguera-Artiaga et al. 2020). Mientras que Lu et al. (2019) y Taha et al. (2020) mencionan que al utilizar el RDC, el metabolismo de las plantas se adapta ante la restricción hídrica, para mantener o mejorar la productividad de las plantas, lo que depende del grado de RDC utilizado. El uso del RDC presenta una eficiencia en el uso del agua en diversos cultivos, principalmente, por que se ha conseguido mejorar la transducción de señales en las células guarda, lo que se traduce en un control estomático optimizado, lo que mejora la fotosíntesis y la transpiración (Chai et al. 2016). En este sentido, los resultados de incremento en la capacidad antioxidante y la actividad de la enzima APX, se relacionan con una mejora en la tasa fotosintética neta mediante la regulación de la conductancia estomática y la actividad de la enzima rubisco (Feng et al. 2020).

La aplicación de los tratamientos con RDC al 50 y $75 \%$ evidenció cambios en el metabolismo antioxidante de las plantas de tomate. Los cuales están 
asociados con la regulación de la homeostasis celular para prevenir un estado de estrés oxidativo, por lo que el metabolismo antioxidante de las plantas es clave en la adaptación de las plantas de tomate bajo RDC. El RDC es una estrategia de gran importancia que se pudiera aplicar en diversos cultivos a nivel nacional, lo que tendría un impacto considerable en el ahorro del agua de riego.

\section{AGRADECIMIENTOS}

Al Consejo Nacional de Ciencia y Tecnología (CONACYT) por la beca otorgada para realizar el Doctorado en Agricultura Protegida en la Universidad Autónoma Agraria Antonio Narro. Saltillo, México.

\section{LITERATURA CITADA}

Agami RA, Alamri SA, Abd El-Mageed TA, Abousekken MSM, Hashem M (2018) Role of exogenous nitrogen supply in alleviating the deficit irrigation stress in wheat plants. Agricultural Water Management 210: 261270.

Bartoli CG, Buet A, Grozeff GG, Galatro A, Simontacchi M (2017) Ascorbate-glutathione cycle and abiotic stress tolerance in plants. In: Hossain MA, Munné-Bosch S, Burritt DJ, Diaz-Vivancos P, Fujita M, Lorence A (eds.) Ascorbic acid in plant growth, development and stress tolerance. Springer. Switzerland. pp: 177-200.

Bigurra-Alzati CA, Ortiz-Gómez R, Vázquez-Rodríguez GA, López-León LD, Lizárraga-Mendiola L (2021) Water conservation and green infrastructure adaptations to reduce water scarcity for residential areas with semiarid climate: Mineral de la reforma, Mexico. Water 13(1): 45. DOI: 10.3390/w13010045.

Bradford MM (1976) A rapid and sensitive method for the quantitation of microgram quantities of protein utilizing the principle of protein-dye binding. Analytical Biochemistry 72: 248-254.

Cansev A, Gulen H, Eris A (2011) The activities of catalase and ascorbate peroxidase in olive (Olea europaea L. cv. Gemlik) under low temperature stress. Horticulture Environment and Biotechnology 52: 113-120.

Chai Q, Gan Y, Turner NC, Zhang RZ, Yang C, Niu Y, Siddique KHM (2014) Water-saving innovations in Chinese agriculture. Advances in Agronomy 126: 147-197.

Chai Q, Gan Y, Zhao C, Xu HL, Waskom R M, Niu Y, Siddique KH (2016) Regulated deficit irrigation for crop production under drought stress. A review. Agronomy for Sustainable Development 36(1): 3. DOI: 10.1007/ s13593-015-0338-6.

Corpas FJ, Del Río LA, Palma JM (2019) Plant peroxisomes at the crossroad of $\mathrm{NO}$ and $\mathrm{H}_{2} \mathrm{O}_{2}$ metabolism. Journal of Integrative Plant Biology 61: 803-816.

Csepregi K, Neugart S, Schreiner M, Hideg É (2016). Comparative evaluation of total antioxidant capacities of plant polyphenols. Molecules 21(2): 208. DOI: 10.3390/molecules21020208.

Denaxa NK, Damvakaris T, Roussos PA (2020) Antioxidant defense system in young olive plants against drought stress and mitigation of adverse effects through external application of alleviating products. Scientia Horticulturae 259: 108812. DOI: 10.1016/j.scienta.2019.108812.

Dvorak P, Krasylenko Y, Zeiner A, Samaj J, Takac T (2020) Signaling toward ROS-scavenging enzymes in plants. Frontiers in Plant Science 11: 2178. DOI: 10.3389/fpls.2020.618835.

FAO. 2016. AQUASTAT database. http://www.fao.org/nr/water/ aquastat/data/. Fecha de consulta: 18 de enero de 2021. 
Feng BH, Li GY, Islam M, Fu WM, Zhou YQ, Chen TT, Fu GF (2020) Strengthened antioxidant capacity improves photosynthesis by regulating stomatal aperture and ribulose-1, 5-bisphosphate carboxylase/oxygenase activity. Plant Science 290: 110245. DOI: 10.1016/j.plantsci.2019.110245.

Gupta AK, Kaur K, Kaur N (2011) Stem reserve mobilization and sink activity in wheat under drought conditions. American Journal of Plant Sciences 2: 70-77.

Haida Z, Hakiman M (2019) A comprehensive review on the determination of enzymatic assay and nonenzymatic antioxidant activities. Food Science \& Nutrition 7: 1555-1563.

He Y, Zhou J, Shan L, Meng X (2018) Plant cell surface receptor-mediated signaling-a common theme amid diversity. Journal of Cell Science 131: 1-11. DOI: 10.1242/jcs.209353.

Koffler BE, Luschin-Ebengreuth N, Stabentheiner E, Müller M, Zechmann B (2014) Compartment specific response of antioxidants to drought stress in Arabidopsis. Plant Science 227: 133-144.

Laxa M, Liebthal M, Telman W, Chibani K, Dietz KJ (2019) The role of the plant antioxidant system in drought tolerance. Antioxidants 8: 94. DOI: 10.3390/antiox8040094.

Leng X, Wang H, Zhang S, Qu C, Yang C, Xu Z, Liu G (2021) Identification and characterization of the APX gene family and its expression pattern under phytohormone treatment and abiotic stress in Populus trichocarpa. Genes 12(3): 334. DOI: 10.3390/genes12030334.

Leung DW (2018) Studies of catalase in plants under abiotic stress. In: Gupta D, Palma J, Corpas F (eds) Antioxidants and antioxidant enzymes in higher plants. Springer, Cham. Switzerland. pp: 27-39.

Lu J, Shao G, Cui J, Wang X, Keabetswe L (2019) Yield, fruit quality and water use efficiency of tomato for processing under regulated deficit irrigation: A meta-analysis. Agricultural Water Management 222: 301312.

Meitha K, Pramesti Y, Suhandono S (2020) Reactive oxygen species and antioxidants in postharvest vegetables and fruits. International Journal of Food Science 2020: 8817778. DOI: 10.1155/2020/8817778.

Melandri G, Abdelgawad H, Riewe D, Hageman JA, Asard H, Beemster GTS, Kadam N, Jagadish K, Altmann T, Ruyter-Spira C, Bouwmeester H (2019) Biomarkers for grain yield stability in rice under drought stress. Journal of Experimental Botany 71(2): 669-683.

Nahar K, Hasanuzzaman M, Alam MM, Fujita M (2015) Roles of exogenous glutathione in antioxidant defense system and methylglyoxal detoxification during salt stress in mung bean. Biologia Plantarum 59: 745-756.

Nakano Y, Asada K (1987) Purification of ascorbate peroxidase in spinach chloroplasts; its inactivation in ascorbatedepleted medium and reactivation by monodehydroascorbate radical. Plant and Cell Physiology 28: 131140.

Nangare DD, Singh Y, Kumar PS, Minhas PS (2016) Growth, fruit yield and quality of tomato (Lycopersicon esculentum Mill.) as affected by deficit irrigation regulated on phenological basis. Agricultural Water Management 171: 73-79.

Nawaz F, Ahmad R, Ashraf MY, Waraich EA, Khan SZ (2015) Effect of selenium foliar spray on physiological and biochemical processes and chemical constituents of wheat under drought stress. Ecotoxicology and Environmental Safety 113: 191-200.

Noguera-Artiaga L, Sánchez-Bravo P, Hernández F, Burgos-Hernández A, Pérez-López D, Carbonell-Barrachina ÁA (2020) Influence of regulated deficit irrigation and rootstock on the functional, nutritional and sensory quality of pistachio nuts. Scientia Horticulturae 261: 108994. DOI: 10.1016/j.scienta.2019.108994. 
Qi J, Song C P, Wang B, Zhou Kangasjärvi J, Zhu J K, Gong Z (2018) Reactive oxygen species signaling and stomatal movement in plant responses to drought stress and pathogen attack. Journal of Integrative Plant Biology 60: 805-826.

Rady MOA, Semida WM, Howladar SM, Abd El-Mageed TA (2020) Raised beds modulate physiological responses, yield and water use efficiency of wheat (Triticum aestivum $\mathrm{L}$ ) under deficit irrigation. Agricultural Water Management 245: 106629. DOI: 10.1016/j.agwat.2020.106629.

Re R, Pellegrini N, Proteggente A, Pannala A, Yang M, Rice-Evans C (1999) Antioxidant activity applying an improved ABTS radical cation decolorization assay. Free Radical Biology and Medicine 26: 1231-1237.

Ren R, Li Z, Zhang L, Zhou H, Jiang X, Liu Y (2020) Enzymatic and nonenzymatic antioxidant systems impact the viability of cryopreserved Paeonia suffruticosa pollen. Plant Cell, Tissue and Organ Culture 144: 233-246.

Saed-Moucheshi A, Shekoofa A, Pessarakli M (2014) Reactive oxygen species (ROS) generation and detoxifying in plants. Journal of Plant Nutrition 37: 1573-1585.

Segura-Castruita MÁ, Ramírez-Seañez AR, García-Legaspi G, Preciado-Rangel P, García-Hernández JL, YescasCoronado P, Fortis-Hernández M, Orozco-Vidal JA, Montemayor-Trejo JA (2011) Desarrollo de plantas de tomate en un sustrato de arena-pómez con tres diferentes frecuencias de riego. Revista Chapingo Serie Horticultura 17: 25-31.

Steiner AA (1961) A universal method for preparing nutrient solutions of a certain desired composition. Plant and Soil 15: 134-154.

Shukla PS, Mantin EG, Adil M, Bajpai S, Critchley AT, Prithiviraj B (2019) Ascophyllum nodosum-based biostimulants: Sustainable applications in agriculture for the stimulation of plant growth, stress tolerance, and disease management. Frontiers in Plant Science 10: 655. DOI: 10.3389/fpls.2019.00655.

Soureshjani HK, Nezami A, Kafi M, Tadayon M (2019) Responses of two common bean (Phaseolus vulgaris L.) genotypes to deficit irrigation. Agricultural Water Management 213: 270-279.

Sweet SK, Wolfe DW, DeGaetano A, Benner R (2017) Anatomy of the 2016 drought in the Northeastern United States: Implications for agriculture and water resources in humid climates. Agricultural and Forest Meteorology 247: 571-581.

Taha RS, Alharby HF, Bamagoos AA, Medani RA, Rady MM (2020) Elevating tolerance of drought stress in Ocimum basilicum using pollen grains extract; a natural biostimulant by regulation of plant performance and antioxidant defense system. South African Journal of Botany 128: 42-53.

Toor MD, Adnan M, Javed MS, Habibah UE, Arshad A, Mughees M, Ahmad R, Danish M (2020) Foliar application of $\mathrm{Zn}$ : Best way to mitigate drought stress in plants: A review. International Journal of Applied Research 6: 16-20.

Xie X, He Z, Chen N, Tang Z, Wang Q, Cai Y (2019) The roles of environmental factors in regulation of oxidative stress in plant. BioMed Research International 2019: 9732325. DOI: 10.1155/2019/9732325.

Xu B, Gao X, Dong K, Li X, Yang P, Yang T, Feng B (2020) Grain protein content comparison and proteomic analysis of foxtail millet (Setaria italica L.) seed response to different drought stress levels. Acta Physiologiae Plantarum 42: 20. DOI: 10.1007/s11738-019-2999-2.

Xue T, Hartikainen H, Piironen V (2001) Antioxidative and growth-promoting effect of selenium on senescing lettuce. Plant and Soil 237: 55-61. 